\title{
DIVISIBILITY BY 2 OF STIRLING-LIKE NUMBERS
}

\author{
DONALD M. DAVIS
}

(Communicated by Andrew Odlyzko)

\begin{abstract}
We give a characterization of functions of the form $f(n)=$ $\nu(n-E)$, where $\nu(-)$ denotes the exponent of 2 , and $E$ is a 2 -adic integer. We show that it applies to the restriction to even or odd integers of the function $f(n)=\nu\left(a \cdot 5^{n}+b \cdot 3^{n}+c\right)$, with mild restrictions on $a, b$, and $c$. This function is closely related to divisibility of certain Stirling numbers of the second kind.
\end{abstract}

Let $\nu(m)$ denote the exponent of 2 in $m$, and let $\mathbf{N}$ denote the set of nonnegative integers. Our first result is a characterization of a certain class of functions. We think of a 2 -adic integer as a possibly infinite sum of distinct 2-powers.

Theorem 1. Let $f$ be a function $\mathbf{N} \rightarrow \mathbf{N} \cup\{\infty\}$. Then the following are equivalent:

(i) There is a 2-adic integer $E$ such that $f(n)=\nu(n-E)$ for all $n$;

(ii) For all $n$ and $d$,

$$
f\left(n+2^{d}\right) \begin{cases}=d & \text { if } d<f(n) \\ >f(n) & \text { if } d=f(n) \\ =f(n) & \text { if } d>f(n)\end{cases}
$$

(iii) $f$ satisfies

(a) for all $n, f\left(n+2^{f(n)}\right)>f(n)$, and

(b) if $d<f(n)$ and $\alpha$ is odd, then $f\left(n+\alpha 2^{d}\right)=d$.

$E$ is defined by $E=\sum_{i \geq 1} 2^{e_{i}}$ with $e_{i}<e_{i+1}$, where $e_{1}=f(0), E(k)=$ $\sum_{i=1}^{k} 2^{e_{i}}$, and $e_{k+1}=f(E(k))$. Finally, $E$ is finite if and only if $\infty \in f(\mathbf{N})$.

Proof. (i) $\Rightarrow$ (ii): $f(n)$ equals the exponent of the smallest 2-power in which $n$ and $E$ differ. Then $n+2^{f(n)}$ and $E$ agree in that 2-power as well, while $n+2^{d}$ and $E$ first differ at $2^{d}$ if $d<f(n)$ and at $2^{f(n)}$ if $d>f(n)$.

Received by the editors September 29, 1989 and, in revised form, January 11, 1990.

1980 Mathematics Subject Classification (1985 Revision). Primary 11B73.

Key words and phrases. Stirling numbers, divisibility by 2, 2-adic integers.

This work was partially supported by an NSF Research Grant. I would also like to thank MSRI, where some of this work was performed. 
(ii) $\Rightarrow$ (iii): Write $\alpha 2^{d}=2^{d}+\Sigma 2^{e_{i}}$, with $e_{i}>d$. Then $f\left(n+2^{d}\right)=d$, and adding each $2^{e_{i}}$ to the argument does not change the value of $f$.

(iii) $\Rightarrow(\mathrm{i})$ : Define $E$ as in the theorem. Hypothesis (a) applied to $E(i-1)$ guarantees that $e_{i}<e_{i+1}$. For any $n$, let $d=\nu(n-E)$, and choose $k$ so that $e_{k} \leq d<e_{k+1}$. Then $n-E(k)=\alpha 2^{d}$, with $\alpha$ odd, and so we apply (b) with $n=E(k)$, noting that the hypothesis is satisfied since $f(E(k))=e_{k+1}>d$. We deduce $f(n)=f\left(E(k)+\alpha 2^{d}\right)=d$.

Our second result is an application of Theorem 1.

Theorem 2. If $a$ is odd, $b \equiv 2 \bmod 4$, and $a+b+c \equiv 0 \bmod 8$, then there exist 2-adic integers $E$ and $E^{\prime}$ such that

$$
\nu\left(a \cdot 5^{n}+b \cdot 3^{n}+c\right)=2+ \begin{cases}\nu(n-E) & \text { if } n \text { even } \\ \nu\left(n-E^{\prime}\right) & \text { if } n \text { odd } .\end{cases}
$$

Let $g(n)=-2+\nu\left(a \cdot 5^{n}+b \cdot 3^{n}+c\right)$. If $E=\Sigma 2^{e_{i}}$ with $e_{i}<e_{i+1}$ and $E^{\prime}=\Sigma 2^{e_{i}^{\prime}}$ with $e_{i}^{\prime}<e_{i+1}^{\prime}$, then $e_{1}=g(0), e_{k+1}=g(E(k)), e_{1}^{\prime}=0$, and $e_{k+1}^{\prime}=g\left(E^{\prime}(k)\right)$.

Proof. We use the well-known and easily proved fact that

$$
\nu\left(p^{i}-1\right)= \begin{cases}\nu(4 i) & \text { if } p=5, \text { or } p=3 \text { and } i \text { even } \\ 1 & \text { if } p=3 \text { and } i \text { odd }\end{cases}
$$

to observe that if

$$
\nu\left(a \cdot 5^{n}+b \cdot 3^{n}+c\right)=e+2,
$$

and $\alpha$ is odd and $d>0$, then

$$
\nu\left(a \cdot 5^{n+\alpha 2^{d}}+b \cdot 3^{n+\alpha 2^{d}}+c\right) \begin{cases}=d+2 & \text { if } d<e \\ >e+2 & \text { if } d=e \\ =e+2 & \text { if } d>e .\end{cases}
$$

Here we have used the decomposition

$$
a \cdot 5^{n+\alpha 2^{d}}+b \cdot 3^{n+\alpha 2^{d}}+c=2^{e+2} \text { odd }+a 5^{n}\left(5^{\alpha 2^{d}}-1\right)+b 3^{n}\left(3^{\alpha 2^{d}}-1\right) .
$$

Thus $g$ satisfies (a) of Theorem 1(iii) and (b) restricted to $d>0$. This implies that the functions $f_{0}$ and $f_{1}$ defined by

$$
f_{\varepsilon}(n)=g(2 n+\varepsilon)-1, \quad \varepsilon=0,1
$$

satisfy the hypotheses of Theorem 1(iii). Indeed,

$$
\begin{aligned}
f_{\varepsilon}\left(n+2^{f_{\varepsilon}(n)}\right) & =g\left(2 n+2^{f_{\varepsilon}(n)+1}+\varepsilon\right)-1 \\
& =g\left(2 n+2^{g(2 n+\varepsilon)}+\varepsilon\right)-1>g(2 n+\varepsilon)-1=f_{\varepsilon}(n),
\end{aligned}
$$

and if $0 \leq d<f_{\varepsilon}(n)$, then $0<d+1<g(2 n+\varepsilon)$; hence

$$
f_{\varepsilon}\left(n+\alpha 2^{d}\right)=g\left(2 n+\alpha 2^{d+1}+\varepsilon\right)-1=(d+1)-1 .
$$


In order that $f_{\varepsilon}(m) \geq 0$, we need $g(2 m+\varepsilon)>0$, which follows from

$$
g(2 m+\varepsilon)=-2+\nu\left(5^{\varepsilon} a\left(5^{2 m}-1\right)+3^{\varepsilon} b\left(3^{2 m}-1\right)+\left(5^{\varepsilon} a+3^{\varepsilon} b+c\right)\right),
$$

since each of the three terms is divisible by 8 , the last since the hypotheses imply $5 a+3 b+c \equiv 0 \bmod 8$.

Thus by Theorem $1, f_{\varepsilon}(n)=\nu\left(n-E_{\varepsilon}\right)$ for some 2-adic $E_{\varepsilon}$, and

$$
g(2 n)=\nu\left(n-E_{0}\right)+1=\nu(2 n-E) \text { with } E=2 E_{0},
$$

and

$$
g(2 n+1)=\nu\left(n-E_{1}\right)+1=\nu\left(2 n+1-E^{\prime}\right) \text { with } E^{\prime}=2 E_{1}+1 .
$$

Similar manipulations imply that $E$ and $E^{\prime}$ satisfy the asserted defining property.

We remark that if $\nu(a+b+c)=v<3$, then $\nu\left(a 5^{n}+b 3^{n}+c\right)=v$ for all even $n$, and if $\nu(5 a+3 b+c)=v^{\prime}<3$, then $\nu\left(a 5^{n}+b 3^{n}+c\right)=v^{\prime}$ for all odd $n$. This is immediate from (3). If $a+b$ is even, $a+b+c \equiv 0 \bmod 8$, and $5 a+3 b+c \equiv 0 \bmod 8$, then $\nu\left(a \cdot 5^{n}+b \cdot 3^{n}+c\right)$ is more complicated.

This work was motivated by a desire to determine the exponent of 2 in some Stirling numbers of the second kind. These exponents are important in algebraic topology [3-5]. We take as our definition

$$
S(n, k)=\frac{1}{k !} \sum_{i=0}^{k}(-1)^{i}\left(\begin{array}{c}
k \\
i
\end{array}\right)(k-i)^{n}, 1 \leq k \leq n .
$$

See [2] for other formulas and combinatorial descriptions. In particular,

$$
S(n, 5)=\frac{1}{5 !}\left(5^{n}-5 \cdot 4^{n}+10 \cdot 3^{n}-10 \cdot 2^{n}+5\right) .
$$

One would expect the second and fourth terms to be much more highly 2 divisible than the combination of the others, in which case $\nu(S(n, 5))$ would equal $-1+g(n)$, where

$$
g(n)=-2+\nu\left(5^{n}+10 \cdot 3^{n}+5\right) .
$$

Theorem 2 applies to this $g$, and a REDUCE program easily calculates the values of the exponents which are less than 100 to be $e_{i}: 2,3,4,7,12,16,17,18,19,21,22,23,25,26,28,29,30,31,34$, $38,41,42,45,50,51,52,53,55,57,58,60,61,62,63,64,66,67$, $71,73,74,75,76,77,78,79,80,81,83,87,91,94,97,98,99$. $e_{i}^{\prime}: 0,1,2,3,4,8,11,14,16,19,20,25,27,28,29,30,35,37,39,40$, $44,47,48,50,53,54,57,58,60,61,62,66,68,69,70,71,73,76$, $78,79,83,85,89,91,94$.

Now, if $E$ is defined from (4) as in Theorem 2, so that the $e_{i}$ 's less than 100 are as above, then

$$
\nu(S(n, 5))=-1+\nu(n-E) \text { for even } n, \text { provided } \nu(n-E)<n-1,
$$


and similarly for odd $n$. The first failure of this would be for $n$ equal to the smallest $E(k)$ such that $e_{k+1} \geq E(k)-1$. It can be observed that this never happens for $5 \leq n<2^{100}$, and for it to happen subsequently would require the unlikely occurrence of more than $2^{94}$ consecutive 0 's in the binary expansion of $E$ or $E^{\prime}$.

It is then a simple matter to read off $\nu(S(n, 5))$ for $n$ even and $n<2^{100}$ from the smallest 2-power in which $n$ differs from $E$, as determined from the list of $e_{i}$ 's, and similarly for $n$ odd. For example, since

$$
1989=2^{0}+2^{2}+2^{6}+2^{7}+2^{8}+2^{9}+2^{10}
$$

we obtain $\nu(S(1989,5))=-1+\nu\left(1989-E^{\prime}\right)=0$.

A similar discussion to all of this applies to $S(n, 6)$. If $k<5$, then $\nu(S(n, k))=0$ or 1 depending on the parity of $n$, and for $k>7, \nu(S(n, k))$ is somewhat more complicated to analyze. In work [1] stimulated by an earlier version of this paper, Francis Clarke has generalized this work in a number of directions (larger $k$, odd primes, and a more general context). I thank him for several useful comments on this work.

\section{REFERENCES}

1. F. Clarke, Hensel's lemma and the divisibility by primes of Stirling numbers of the second kind (to appear).

2. L. Comtet, Advanced combinatorics, Reidel, Boston, 1974.

3. M. Crabb and K. Knapp, The Hurewicz map on stunted complex projective spaces, Amer. J. Math. 110 (1988), 783-809.

4. A. T. Lundell, Generalized e-invariants and the numbers of James, Quart. J. Math. Oxford 25 (1974), 427-440.

5. __ A divisibility property for Stirling numbers, J. Number Theory 10 (1978), 35-54.

Department of Mathematics, Lehigh University, Bethlehem, Pennsylvania 18015 